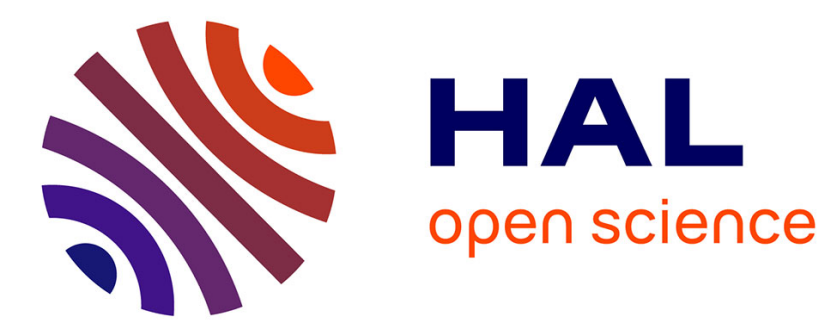

\title{
Strategies for the Controlled Covalent Double Functionalization of Graphene Oxide
}

Isabella Anna Vacchi, Shi Guo, Jesus Raya, Alberto Bianco, Cecilia

Ménard-Moyon

\section{- To cite this version:}

Isabella Anna Vacchi, Shi Guo, Jesus Raya, Alberto Bianco, Cecilia Ménard-Moyon. Strategies for the Controlled Covalent Double Functionalization of Graphene Oxide. Chemistry, 2020, 10.1002/chem.201905785. hal-02926641

\section{HAL Id: hal-02926641 \\ https://hal.science/hal-02926641}

Submitted on 31 Aug 2020

HAL is a multi-disciplinary open access archive for the deposit and dissemination of scientific research documents, whether they are published or not. The documents may come from teaching and research institutions in France or abroad, or from public or private research centers.
L'archive ouverte pluridisciplinaire HAL, est destinée au dépôt et à la diffusion de documents scientifiques de niveau recherche, publiés ou non, émanant des établissements d'enseignement et de recherche français ou étrangers, des laboratoires publics ou privés. 


\title{
Strategies for the Controlled Covalent Double Functionalization of Graphene Oxide
}

\author{
Isabella A. Vacchi, ${ }^{[a]}$ Shi Guo, ${ }^{[a]}$ Jésus Raya,${ }^{[b]}$ Alberto Bianco, ${ }^{[a]}$ and Cécilia Ménard-Moyon*[a] \\ ${ }^{[a]}$ CNRS, Immunology, Immunopathology and Therapeutic Chemistry, UPR 3572, University of \\ Strasbourg, ISIS, 67000 Strasbourg (France) \\ ${ }^{[b]}$ Membrane Biophysics and NMR, Institute of Chemistry, UMR 7177, University of Strasbourg, \\ Strasbourg (France)
}

E-mail: $\underline{\text { c.menard@ibmc-cnrs.unistra.fr and a.bianco@ibmc-cnrs.unistra.fr }}$

\begin{abstract}
Graphene oxide (GO) is a versatile platform with unique properties that have found broad applications in the biomedical field. Double functionalization is a key aspect to design multifunctional GO with combined imaging, targeting, and therapeutic properties. Compared to non-covalent functionalization, covalent strategies lead to GO conjugates with a higher stability in biological fluids. However, only a few double covalent functionalization approaches have been developed so far. The complexity of GO makes the derivatization of the oxygenated groups difficult to control. We investigated the combination of a nucleophilic epoxide ring opening with the derivatization of the hydroxyl groups via esterification or the Williamson reaction. The conditions were selective and mild, thus preserving the structure of GO. Our strategy of double functionalization holds a high potential for different applications where the derivatization of GO with different molecules is needed, especially in the biomedical field.
\end{abstract}

\section{Keywords}

Carbon materials; Esterification; Protecting groups; Surface chemistry; Williamson reaction 


\section{Introduction}

Graphene oxide (GO) has been extensively investigated in many fields, ${ }^{[1,2]}$ in particular in nanomedicine because of its excellent aqueous processability, amphiphilicity and the possibility of surface functionalization. ${ }^{[3-5]}$ In its reduced form it displays thermal and electrical conductivity that can be exploited for the development of biosensors. ${ }^{[6,7]}$ The large surface area and the chemical structure of GO enable several chemical modifications, ${ }^{[8-10]}$ making it an excellent platform for multifunctionalization through covalent and/or non-covalent approaches. Covalent functionalization is more stable compared to non-covalent interactions and this is preferable for biomedical applications as there is a risk of release of the bioactive molecules adsorbed onto the GO surface in the biological media. ${ }^{[11]}$ Multifunctionalization strategies allow to combine the properties of several molecules with those of GO. Until now, only few covalent double functionalization strategies of GO have been reported. Even if GO is composed of different functional groups, mainly epoxides, hydroxyls, and a little amount of carboxylic acids, ${ }^{[12]}$ most approaches mainly exploited the same functional groups on the surface of GO or different functional groups without any selectivity. Few research groups developed a double functionalization process targeting selectively two functional groups already present on the surface of GO such as the epoxides or hydroxyls, and the carboxylic acids. ${ }^{[13-16]}$ For instance, Imani et al. prepared multifunctionalized GO by derivatization of the hydroxyls using chloroacetic acid and sodium hydroxide, followed by amidation of the carboxylated GO with a mixture of polyethylene glycol diamine and octa-arginine. ${ }^{[13]}$ Rezaei et al. performed the Ugi reaction on carboxylated GO using cyclohexylisocyanide, an aldehyde and an amine derivative. ${ }^{[14]}$ In both studies the hydroxyls on the basal plane and the $\mathrm{COOH}$ groups at the edges of $\mathrm{GO}$ were exploited for functionalization. The combination of epoxide ring opening using an amine derivative followed by amidation of the carboxylic acids using a second amine-bearing compound has also been reported. ${ }^{[15]}$ In another approach Al-Jamal and coworkers synthesized GO doubly functionalized with an azide and an alkyne moiety. ${ }^{[16]}$ Sodium azide reacted as nucleophile to open the epoxides and the alkyne moiety was subsequently introduced by Steglich esterification of the carboxyl groups. However, GO had to be pre-treated with $m$-chloroperoxybenzoic acid before functionalization to increase the amount of epoxides on its surface. In addition, all these strategies involve a reaction exploiting the $\mathrm{COOH}$ groups at the edges of $\mathrm{GO}$, and we previously demonstrated that they are present only in low amount, ${ }^{[12]}$ thus resulting in low levels of functionalization. Recently, we reported a simple and versatile strategy for the covalent double functionalization of GO based on the combination of epoxide opening by a thiol derivative and a Michael addition of the hydroxyls with benzoquinone. ${ }^{[17]}$ In this context, we wish to extend the possibilities of double functionalization of GO through selective derivatization of the epoxides and hydroxyls. For this purpose, we designed a strategy combining a 
nucleophilic epoxide opening reaction with the derivatization of the hydroxyl groups by esterification or etherification (Williamson reaction). We used mild conditions (room temperature and mild base) to preserve the structure and properties of GO. Indeed, harsh conditions such as heating or the use of strong bases often cause partial reduction of GO, thus drastically decreasing its water dispersibility. ${ }^{[18-21]}$

\section{Results and Discussion}

\section{Combination of epoxide opening and Williamson reaction}

The first double functionalization strategy we investigated was the combination of a nucleophilic epoxide opening and the Williamson reaction. We decided to perform first the opening of the epoxides by an amine derivative because the number of hydroxyl groups available for the subsequent Williamson reaction increases after the epoxide ring opening. The reaction was performed on GO from NanoInnova (named GO-N, see Materials and Methods part in Supporting Information). The Boc mono-protected triethylene glycol (TEG) diamine $\mathbf{1}$ was mixed to a suspension of GO in DMF obtaining GO-OE (OE stands for opening of epoxides) (Scheme 1). ${ }^{[12]}$ For the Williamson reaction GO-OE was mixed with the iodinated derivative 2 and $\mathrm{K}_{2} \mathrm{CO}_{3}$ in DMF leading to GO-OEW (W stands for Williamson reaction). ${ }^{[22]}$ Potassium carbonate is commonly used to perform the Williamson reaction of phenol derivatives and it is mild enough to avoid reduction of GO. ${ }^{[22]}$ In addition, both reactions were performed at room temperature, thus preserving the structure of GO.

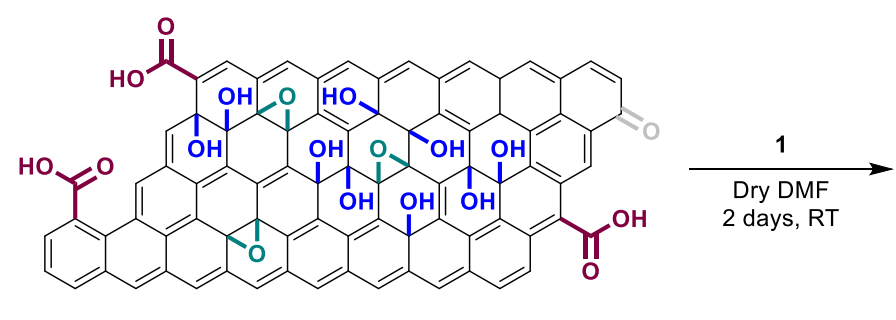

GO-N

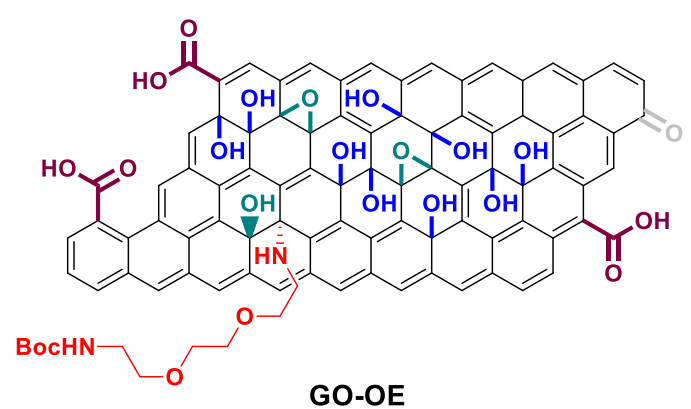

GO-OE
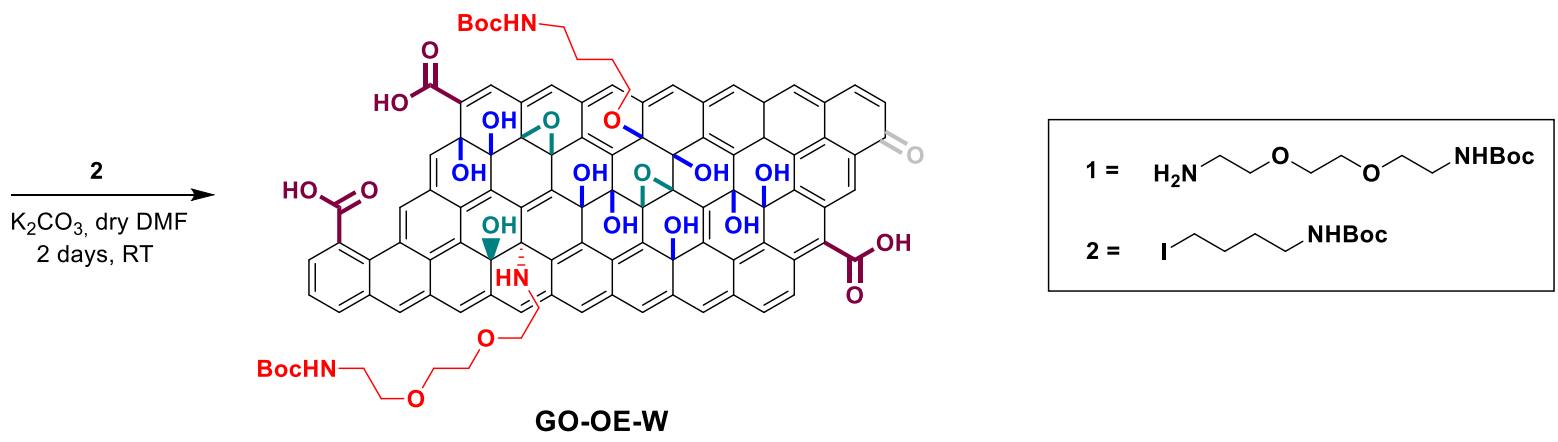

Scheme 1. Double functionalization approach combining the opening of the epoxides and the Williamson reaction (each reaction is shown only on one functional group for clarity reasons). 
GO-N and GO-OE-W were characterized by transmission electron microscopy (TEM) (Figure S1a and $b$ ). The graphene sheets have a lateral dimension of a few micrometers and a wavy shape with folded edges. By comparison to GO-N, we found that the morphology of GO was preserved after the two-step derivatization.

We also characterized GO-OE and GO-OE-W by thermogravimetric analysis (TGA) under inert atmosphere (Figure S2). Because of thermal instability of GO due to labile oxygenated groups that makes the interpretation of the TGA data difficult, ${ }^{[23-25]}$ we also prepared control samples GO-OE_CONT and GO-OE-W_CONT by simply mixing GO-N in similar conditions but without adding 1 and 2 for the control reactions of the epoxide opening and the Williamson reaction, respectively (Scheme S1). Even though the conditions used for the reaction were mild, the control GO samples displayed a higher thermal stability due to some labile functionalities that were removed from the surface of GO. ${ }^{[26-30]}$ As a consequence, TGA is more useful in this case to compare the weight loss between samples of GO already functionalized. Nevertheless, the thermogravimetric curves of the functionalized samples, GO-OE and GO-OE-W, compared to their respective control samples did not show a significantly higher weight loss probably due to low molecular weight of the grafted molecules (Figure S2). However, GO-OE-W displayed a slightly higher weight loss above $300^{\circ} \mathrm{C}$ compared to GO-OE, which is indicative of successful Williamson reaction.

Characterization by X-ray photoelectron spectroscopy (XPS) allowed to determine the percentage of nitrogen introduced on GO-N (Figure S3). Values of 1.45\% and 1.70\% of nitrogen were found for GO-OE and GO-OE-W, respectively, while no nitrogen was found in GO-N (Table S1). There is a significant difference, even if not high, in the nitrogen percentage between the two steps of the double functionalization, indicating that both reactions happened. This result is supported by the XPS high resolution spectra confirming the introduction of the two functional chains. Indeed, in the $\mathrm{O} 1 \mathrm{~s}$ spectra of GO-OE the area percentage of the $\mathrm{C}=\mathrm{O}$ peak that could be assigned to the urethane moiety of the Boc protecting group increases significantly (Figure S3). Unfortunately, the C1s peak does not bring insights on the outcome of the reaction due to the complexity of the deconvolution. In the N1s high resolution spectra of GO-OE and GO-OE-W a main peak centred at 399-400 eV was identified, which can be attributed to the amino and amide groups of $\mathbf{1}$ and $\mathbf{2}$ grafted onto GO. ${ }^{[31]}$ Thus, the XPS analysis confirmed the occurrence of both successive reactions. In addition, the $\mathrm{C} / \mathrm{O}$ ratio did not change much (2.04 for GO-N vs. 2.30 and 2.38 for GO-OE and GO-OE-W, respectively), indicating that the conditions were mild enough to avoid reduction of GO. Finally, the level of functionalization was assessed using a colorimetric test (Kaiser test) that allows to determine the amount of primary amino groups present on GO. The amine loading was 145 and $175 \mu \mathrm{mol} / \mathrm{g}$ after treating GO-OE and GO-OE-W with a solution of $\mathrm{HCl}$ in 1,4-dioxane to cleave the Boc group (Scheme S2, Table S1). 
We estimated that the level of functionalization was one amine every 347 and 287 carbon atoms, respectively (see Table S1 and part 3 in the SI for the explanations on the calculations). It is worth noting that the control samples GO-OE_CONT and GO-OE-W_CONT were also characterized by the Kaiser test after acidic treatment and no significant amine loading was found, which indicates that compounds $\mathbf{1}$ and $\mathbf{2}$ do not adsorb on the surface of GO. This result suggests that the functionalization is certainly covalent in the case of GO-OE and GO-OE-W. In addition, the control sample GOOE_CONT revealed no trace of nitrogen by XPS, showing that there is no residual DMF in the sample. The level of functionalization was $145 \mu \mathrm{mol} / \mathrm{g}$ of GO for the first step (epoxide opening), while the outcome for the Williamson reaction corresponds to the difference between the two loading values $(30 \mu \mathrm{mol} / \mathrm{g})$ (Table S1). Thus, the combination of these two reactions was successful. In our previous work the monofunctionalization of GO-N by the Williamson reaction gave an amine loading of $132 \mu \mathrm{mol} / \mathrm{g} .{ }^{[22]}$ In comparison, the same reaction performed after the opening of the epoxides in the current study is not as efficient as expected. Different reasons could explain the difficulty to reach high loadings for the second reaction, for instance a steric hindrance due to the grafting of a high amount of compound $\mathbf{1}$ on GO and/or the loss of labile hydroxyl groups from the surface of GO during the different reaction steps.

To better investigate the second hypothesis related to the instability of the hydroxyl groups, we performed the double functionalization of GO by inverting the epoxide opening and the Williamson reaction. For this purpose, compound 2 was first added to $\mathbf{G O}-\mathbf{N}$ in the presence of $\mathrm{K}_{2} \mathrm{CO}_{3}$ to get GO-W (Scheme S3). Then, compound 1 reacted with GO-W to open the epoxides giving GO-W-OE. The Kaiser test was performed after Boc deprotection (Table S1 and Scheme S2). The amine loading of GO-W and GO-W-OE was 106 and $145 \mu \mathrm{mol} / \mathrm{g}$, respectively. We could deduce that the loading corresponding to the epoxide opening was only $39 \mu \mathrm{mol} / \mathrm{g}$ in this case. Therefore, by inverting the order of both reactions a slightly lower loading was obtained for the first step (106 vs. $145 \mu \mathrm{mol} / \mathrm{g}$ for epoxide opening), while the loading of the second step was increased by only 9 $\mu \mathrm{mol} / \mathrm{g}$. The functionalized GO samples were also characterized by XPS (Figure S4). For GO-W and GO-W-OE $0.96 \%$ and $1.57 \%$ of nitrogen were measured by XPS, respectively, while $0.6 \% \mathrm{~N}$ was found for the control sample GO-W_CONT, which is lower compared to GO-W (data not shown), indicating that there are probably traces of DMF in the sample. As before, similar changes were identified in the high resolution $\mathrm{O} 1 \mathrm{~s}$ and N1s spectra giving evidence of the introduction of amines protected by the Boc group. Furthermore, the $\mathrm{C} / \mathrm{O}$ ratio was rather similar before and after functionalization (2.04 for GO-N vs. 2.39 and 2.08 for GO-W and GO-W-OE, respectively). The TGA curves of GO-W and GO-W-OE did not show significant differences in comparison with their 
respective control samples GO-W_CONT and GO-W-OE_CONT prepared by simply mixing GON and GO-W_CONT in similar conditions but without compounds 2 and 1, respectively (Figure S5 and Scheme S4). Again, the thermal instability of GO and the low molecular weight of the molecules grafted on GO makes the interpretation of the TGA data complicated. TEM showed that the morphology of the doubly functionalized GO was unaffected after both reactions (Figure S1c).

In this alternative approach the loading for the second reaction was only slightly increased compared to the first strategy. This suggests that, besides the instability of the hydroxyl groups, there must be another reason, such as steric hindrance, that hampers reaching a higher loading for the second step. Therefore, we decided to investigate further the first approach, which was the most efficient based on XPS and the Kaiser test, by optimizing the second step through the derivatization of the hydroxyl groups by esterification instead of the Williamson reaction because of potential higher reactivity.

\section{Epoxide opening followed by esterification}

We repeated the epoxide opening of GO-N by compound $\mathbf{1}$ giving again GO-OE (Scheme S5). The esterification of the hydroxyl groups with Boc-protected aminocaproic acid $\mathbf{3}$ was performed in the presence of 1-ethyl-3-(3-dimethylaminopropyl)carbodiimide (EDC) and 4dimethylaminopyridine (DMAP) as coupling agents at room temperature to get GO-OE-EST (EST stands for esterification). As compounds $\mathbf{1}$ and $\mathbf{3}$ bear a primary amine protected with a Boc group, GO-OE and GO-OE-EST were treated in acid conditions to cleave the Boc moiety (Scheme S2 and S6). The amine loading obtained by the Kaiser test was 179 and $231 \mu \mathrm{mol} / \mathrm{g}$ for GO-OE and GOOE-EST, respectively, confirming the successful introduction of both molecules (Table S2). The epoxide opening led to a loading similar to the value obtained previously (145 $\mu \mathrm{mol} / \mathrm{g}$, Table S1). The level of functionalization for the esterification reaction was $52 \mu \mathrm{mol} / \mathrm{g}$, which is higher compared to the Williamson reaction and the epoxide opening performed as second reaction $(30$ and $39 \mu \mathrm{mol} / \mathrm{g}$, respectively). The level of functionalization for GO-OE-EST was estimated 1 amine every 218 carbon atoms, which is higher compared to GO-OE-W (1 every 287 C) (Table S1 and S2). Therefore, the hydroxyl groups are slightly more reactive towards esterification in comparison to the Williamson reaction. A control reaction was performed by treating GO-N in similar conditions of the epoxide opening but without the addition of compound $\mathbf{1}$ followed by mixing with compound $\mathbf{3}$ in the absence of the coupling agents giving GO-OE_CONT and GO-OE-EST_CONT (Scheme S7). The Kaiser test value obtained for GO-OE-EST_CONT after acidic treatment was negative $(6 \mu \mathrm{mol} / \mathrm{g})$ confirming the absence of $\mathbf{3}$ non-covalently adsorbed on GO.

XPS confirmed that both reactions occurred on the surface of GO-N as an introduction of 1.96 and $2.47 \%$ of nitrogen was detected for GO-OE and GO-OE-EST, respectively (Figure S6), whereas 
only $0.44 \% \mathrm{~N}$ was found for the control sample GO-OE_CONT due to probable traces of residual DMF (data not shown). For this double functionalization strategy, the O1s spectra showed an increase of the $\mathrm{C}=\mathrm{O}$ peak area due to the introduction of both $\mathbf{1}$ and $\mathbf{3}$. The $\mathrm{C} / \mathrm{O}$ ratio slightly increased from 2.04 for GO-N to 2.27 and 2.64 for GO-OE and GO-OE-EST, respectively, indicating that the structure of GO was not affected after functionalization. The N1s high resolution spectrum displayed the typical peak of the amine and amide at $399-400 \mathrm{eV}$, confirming the functionalization. We also performed carbon KLL Auger spectroscopy for all samples (Figure S6i). The analysis of these peaks gives an idea of the quantity of $\mathrm{sp}^{2}$ and $\mathrm{sp}^{3}$ carbon atoms present on GO. ${ }^{[32,33]}$ This estimation is obtained by calculating the distance between the maximum and the minimum binding energy of the first derivative of the C KLL spectra. There was no significant difference in distance among all the samples analyzed, demonstrating that the different reactions did not affect the structure of GO. We can notice that GO-OE-EST displayed closer maximum and minimum values, indicating a slightly more important content of $\mathrm{sp}^{3} \mathrm{C}$ atoms. This could be related to the $\mathrm{sp}^{3} \mathrm{C}$ atoms from the alkyl chains in compounds $\mathbf{1}$ and $\mathbf{3}$.

TGA showed rather similar curves for both GO-OE and GO-OE-EST and their respective controls GO-OE_CONT and GO-OE-EST_CONT, giving no clear evidence of double functionalization of GO (Figure S7).

TEM confirmed the unchanged morphology of the doubly functionalized GO sheets (Figure S1d). Finally, GO-OE-EST was examined by quantitative ${ }^{13} \mathrm{C}$ direct polarization (DP) and cross polarization (CP) magic angle spinning (MAS) solid-state NMR (Figure S8). The peaks at $\sim 60$ and $70 \mathrm{ppm}$ are attributed to the epoxides and hydroxyl groups, respectively, while the peak at $\sim 130 \mathrm{ppm}$ corresponds to the $\mathrm{C}=\mathrm{C}$ bonds. We calculated the percentage of all peaks by integrating each peak after fitting (Table S3a). We estimated that GO-N contains $23 \%$ epoxides, $28 \%$ hydroxyls, and $37 \%$ $\mathrm{C}=\mathrm{C}$ bonds. After the reactions, we noticed the appearance of peaks at 27 and $40 \mathrm{ppm}$ that can be assigned to the Boc protecting group and the methylene moieties of compounds $\mathbf{1}$ and $\mathbf{3}$, respectively. Moreover, the quantitative spectra showed a decrease in the intensity of the peak at $\sim 60 \mathrm{ppm}$, confirming the opening of the epoxide ring (Figure S8, bottom spectrum). Unfortunately, we were not able to detect the peak of the ester function at $\sim 170 \mathrm{ppm}$ probably due to very low intensity.

Taken together, XPS, the Kaiser test, and MAS NMR confirmed the positive outcome of both reactions leading to doubly functionalized GO. Nevertheless, it should be taken into consideration that the loading of the second reaction was still much lower $(52 \mu \mathrm{mol} / \mathrm{g})$ compared to the esterification performed as mono-functionalization of GO-N (165 $\mu \mathrm{mol} / \mathrm{g}$ as published in our previous study). ${ }^{\text {[22] }}$ We think that reasonable explanations for such lower reactivity for the second functionalization reaction could be due to the combination of two phenomena: 1) the instability of some labile hydroxyl 
groups on the surface of GO, and 2) localization confinement of the functional groups on islands that causes a steric hindrance for the second reaction because of the molecules introduced during the first step. Indeed, in the literature an island localization of the oxygenated functional groups on the surface of GO has been reported by many groups. ${ }^{[34-37]}$ Through aberration corrected TEM Erickson et al. observed that there are graphitic regions in GO up to $8 \mathrm{~nm}^{2}$ and that the oxidized regions exhibit no order and have a minimal $\mathrm{sp}^{2}$ bonding character. ${ }^{[37]}$ This type of structure was also confirmed by Zhou et al. using statistical calculations. ${ }^{[36]}$ Their study showed that the oxygen functionalities tend to diffuse, agglomerate, and form highly oxidized domains on GO.

We decided to extend the most efficient strategy of double functionalization, based on the combination of epoxide opening and esterification, to another source of GO (from Grupo Antolin, named GO-A, see Supporting Information). This sample contains less aggregated sheets and it is much more dispersible in water compared to GO from NanoInnova. As a consequence, GO-A is more suitable for applications requiring high water dispersibility such as those in nanomedicine. In this case, we used two orthogonal protecting groups, namely Boc and 1-(2-nitrophenyl)ethyl carbamate, ${ }^{[38-40]}$ to protect the two primary amines grafted onto the surface of GO in order to give the possibility of further selective amine derivatization. Reducing and drastic conditions to remove protecting groups usually affect GO and this is the reason why they should be avoided. In this context, photocleavable moieties are attractive alternative protecting groups.

We first performed the epoxide opening reaction by adding compound $\mathbf{1}$ to GO-A giving $\mathbf{G O}_{\mathbf{A}}-\mathbf{O E}$ (Scheme 2). Afterwards, compound 5 (synthesized in two steps via intermediate 4, Scheme S8) was added to $\mathbf{G O}_{\mathrm{A}}-\mathbf{O E}$ in the presence of EDC and DMAP as coupling agents to obtain $\mathbf{G O}_{\mathrm{A}}-\mathbf{O E}-\mathbf{E S T}$ (Scheme 2). 


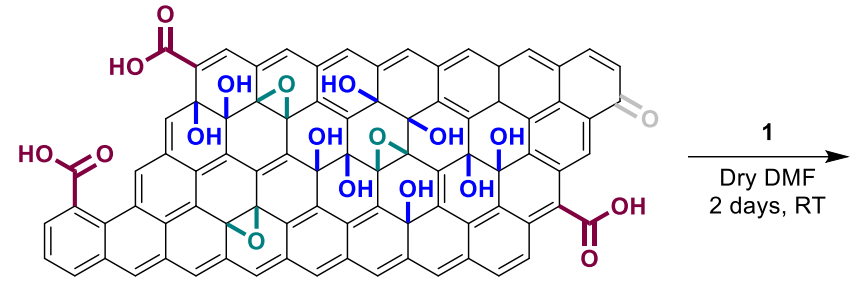

GO-A

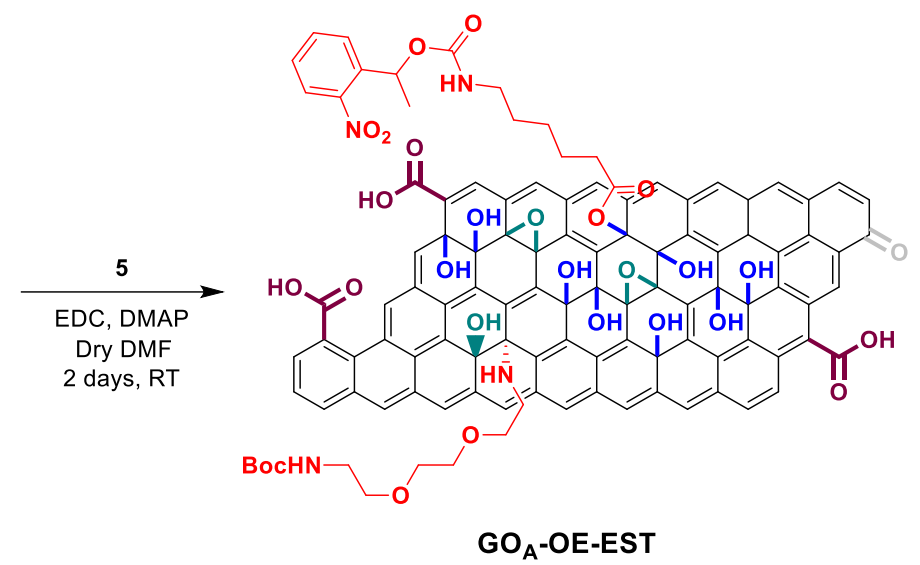

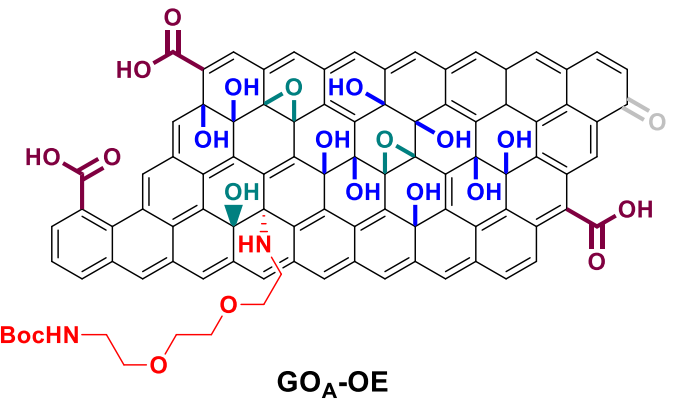

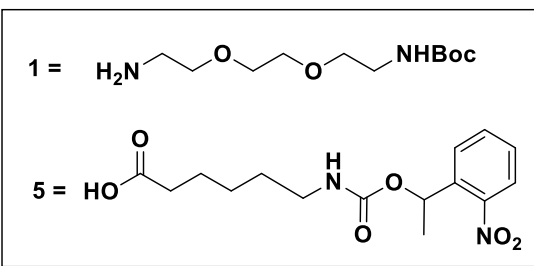

Scheme 2. Double functionalization of GO performed by combining the epoxide opening with esterification on GO-A (each reaction is shown only on one functional group for clarity reasons).

The final conjugate was first treated with $\mathrm{HCl}$ in 1,4-dioxane to cleave the Boc group and then irradiated at $365 \mathrm{~nm}$ to cleave the 1-(2-nitrophenyl)ethyl carbamate group (Scheme S9). The level of functionalization was determined by the Kaiser test giving an average amine loading of $34 \mu \mathrm{mol} / \mathrm{g}$ and $71 \mu \mathrm{mol} / \mathrm{g}$ after Boc deprotection and photocleavage of $\mathbf{G O}_{\mathrm{A}}-\mathbf{O E}-\mathbf{E S T}$, respectively. (Table S2). We deduced that the level of functionalization for the esterification reaction was $37 \mu \mathrm{mol} / \mathrm{g}$, which is consistent with the value obtained when the photocleavage was performed before Boc deprotection (41 $\mu \mathrm{mol} / \mathrm{g}$ ). The reactivity of GO-A is relatively similar to that of GO-N towards esterification (52 $\mu \mathrm{mol} / \mathrm{g})$. Nevertheless, we do not have clear explanations for the lower amine loading obtained for the epoxide opening reaction on GO-A compared to GO-N. A possible reason may be a batch-tobatch variability.

XPS confirmed the introduction of the two chains as $1.72 \%$ and $3.39 \%$ of nitrogen was found for the first and the second step, respectively (Figure 1), while only $0.46 \% \mathrm{~N}$ was obtained for the control sample GOA-OE_CONT (data not shown). Moreover, in the N1s high resolution spectra it was possible to identify the characteristic peak for the nitro moiety of the 1-(2-nitrophenyl)ethyl group at $405.7 \pm 0.2 \mathrm{eV}$ in addition to the peak at $399-400 \mathrm{eV}$ corresponding to amines and amides (Figure $1 \mathrm{~h}$ ). As previously mentioned, because of the complexity of the deconvolution of the $\mathrm{C} 1 \mathrm{~s}$ peak, no conclusion on the success of the reactions can be drawn from the C1s spectra (Figure S9). 
Nevertheless, an increase in the $\mathrm{C}=\mathrm{O}$ peak was detected in the $\mathrm{O} 1 \mathrm{~s}$ high resolution spectra, confirming the introduction of compounds $\mathbf{1}$ and $\mathbf{5}$ (Figure 1d,g). The first derivative of the C KLL spectra showed no significant difference in distance for the derivatized GO samples compared to GO-A, indicating that the double functionalization strategy preserved the $\pi-\pi$ conjugated structure of GO (Figure S9d). This was also confirmed by the small increase of the C/O ratio (2.33 for GO-A vs. 2.47 for $\mathbf{G O}_{\mathrm{A}}-\mathrm{OE}$ and $2.75 \mathrm{GO}_{\mathrm{A}}-\mathrm{OE}-\mathbf{E S T}$, respectively).
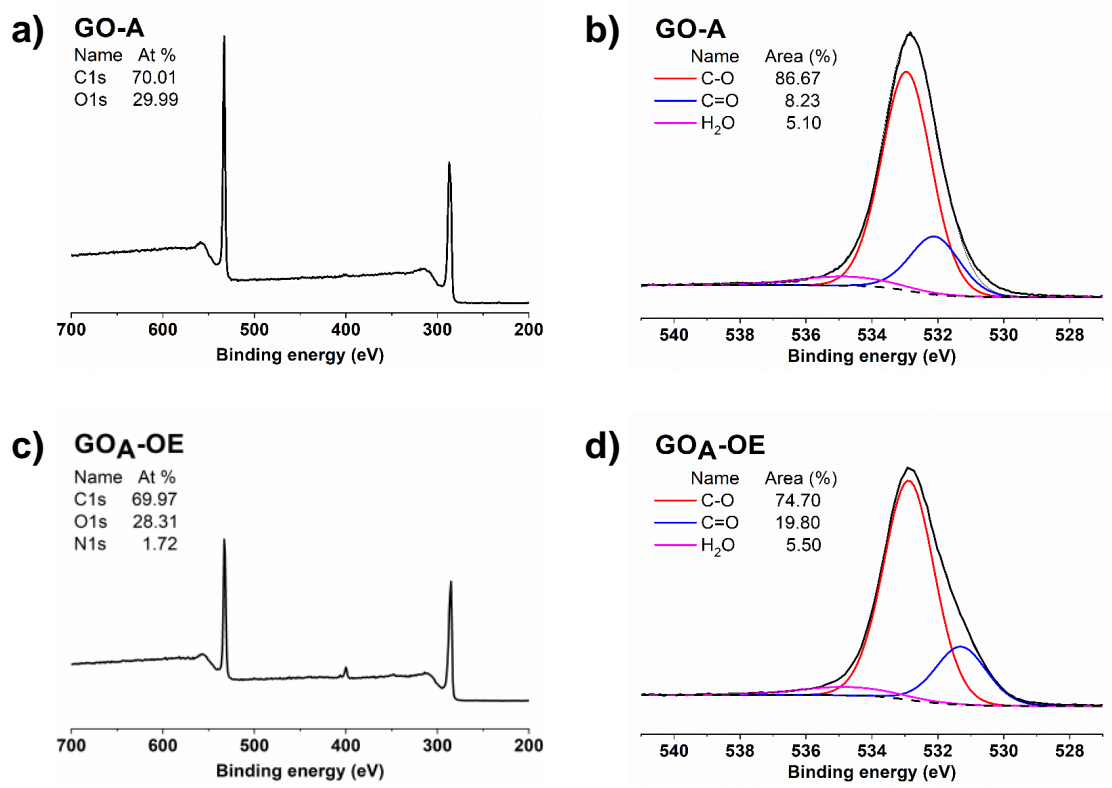

e) $\mathrm{GO}_{\mathrm{A}}-\mathrm{OE}$
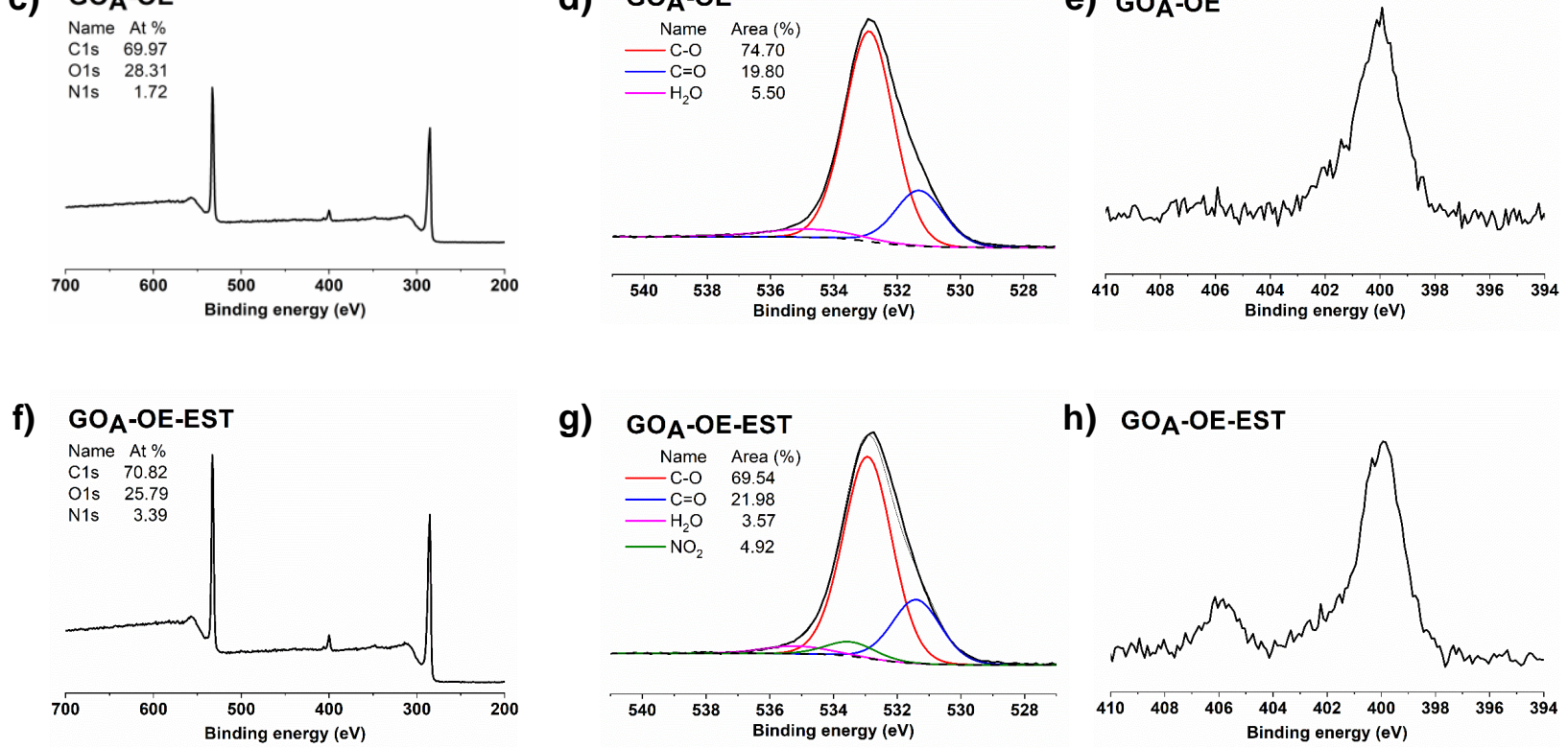

h) $\mathrm{GO}_{\mathrm{A}}$-OE-EST

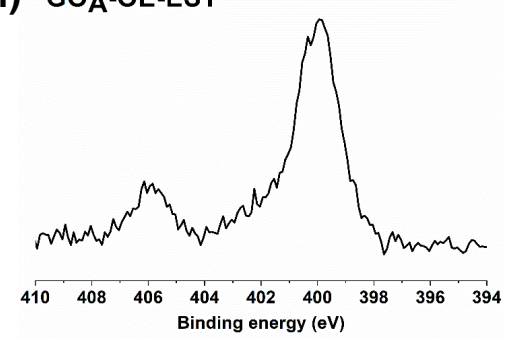

Figure 1. (a) (c) (f) XPS survey spectra of GO-A, GOA-OE, and GOA-OE-EST; (b) (d) (g) O1s of GO-A, GOA-OE, and GOA-OE-EST; (e) (h) N1s spectra of GOA-OE and GOA-OE-EST.

The thermogravimetric curves of GOA-OE and GOA-OE-EST clearly showed different thermal profiles compared with their respective controls GOA-OE_CONT and GOA-OE-EST_CONT (Figure 2 and Scheme S10). We observed a higher weight loss for GOA-OE-EST compared to $\mathbf{G O}_{\mathrm{A}}$ $\mathbf{O E}$ and the control samples in the temperature range of $400-800^{\circ} \mathrm{C}$, which is indicative of a higher amount of functional groups, confirming that GO was successfully doubly functionalized. The first derivatives of the TGA curves (DTG) are shown in Figure S10 and the peaks indicate the temperature of the maximum rate of change on the weight loss curves (also known as the inflection points). The DTG curves allowed comparing precisely the thermal profiles of the different samples. The first 
main slope in the TGA of $\mathbf{G O}_{\mathrm{A}}-\mathbf{O E}$ occurred at lower temperature (centered at $184^{\circ} \mathrm{C}$ ) compared with GO-A $\left(220^{\circ} \mathrm{C}\right)$ and $\mathbf{G O}_{\mathrm{A}}-\mathbf{O E} \_\mathbf{C O N T}\left(200^{\circ} \mathrm{C}\right)$. It can be explained by the introduction of the TEG chain covalently grafted on GO-A. The TGA of compound $\mathbf{1}$ shows that it was degraded at around $184^{\circ} \mathrm{C}$, while the 1-(2-nitrophenyl)ethyl derivative 5 was degraded at $262^{\circ} \mathrm{C}$. After esterification the main slope occurred at higher temperature $\left(196^{\circ} \mathrm{C}\right)$, which can be attributed to the grafting of compound 5. In addition, the weight loss between 50 and $220^{\circ} \mathrm{C}$ was lower after the second reaction, which can be explained by a lower amount of $\mathrm{OH}$ groups available on the GO surface due to the derivatization of the hydroxyls. An additional peak appeared at $370^{\circ} \mathrm{C}$ in the DTG of both GO $_{\mathrm{A}}-\mathrm{OE}$ and $\mathbf{G O}_{\mathrm{A}}-\mathrm{OE}-\mathbf{E S T}$, whereas it was absent in the DTG of the control samples, which could be assigned to the presence of the functional groups on GO (Figure S10). Overall, TGA confirmed that GO-A was successfully doubly functionalized via combined epoxide ring opening and esterification reactions.

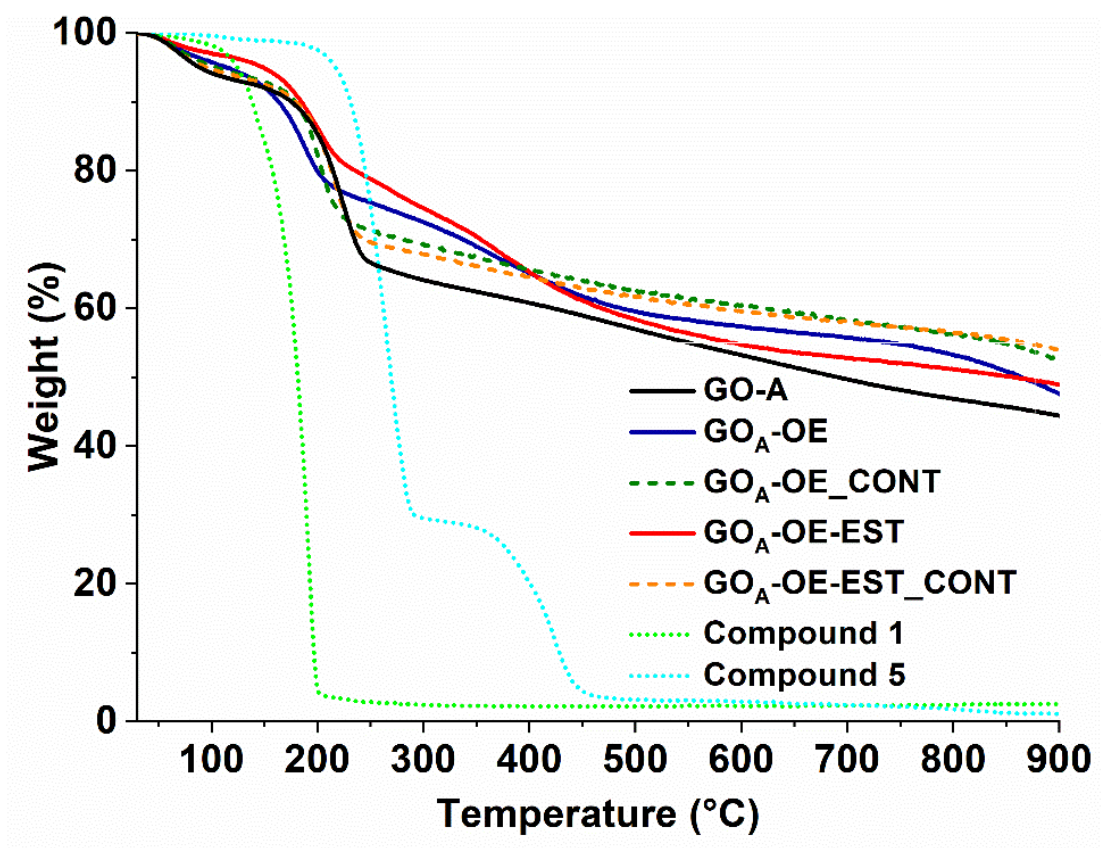

Figure 2. TGA of GO-A, GOA-OE, GOA-OE-EST, GOA-OE_CONT, GOA-OE-EST_CONT, and compounds $\mathbf{1}$ and $\mathbf{5}$ performed under inert atmosphere.

TEM showed that the morphology of GO-A was not affected by the different treatments (Figure S1e,f).

Finally, GOA-OE-EST was characterized by $\mathrm{CP}$ and quantitative DP MAS ${ }^{13} \mathrm{C}$ NMR (Figure 3 ). We estimated that GO-A is constituted of $15 \%$ epoxides, $25 \%$ hydroxyls, and $45 \% \mathrm{C}=\mathrm{C}$ bond (Table S3b). Compared to GO-A, the peak attributed to the epoxides at $60 \mathrm{ppm}$ decreased in the DP spectrum because of the introduction of 1 (Figure 3, bottom spectrum), as previously observed in the case of 
GO-N. At about 165 ppm we were delighted to see the peak of the ester group in both DP and CP spectra, confirming the introduction of $\mathbf{5}$. Beside the ester peak, we could also observe the appearance of other new peaks that confirmed the introduction of both chains, in particular the peak of the carbamate of the Boc group and 1-(2-nitrophenyl)ethyl moiety at $156 \mathrm{ppm}$, the C-N bond of $\mathbf{1}$ and $\mathbf{5}$ at 39 ppm, as well as the $\mathrm{CH}_{3}$ of $\mathbf{1}$ and $\mathrm{CH}_{2}$ from the aliphatic chain of $\mathbf{5}$ at 26 ppm.

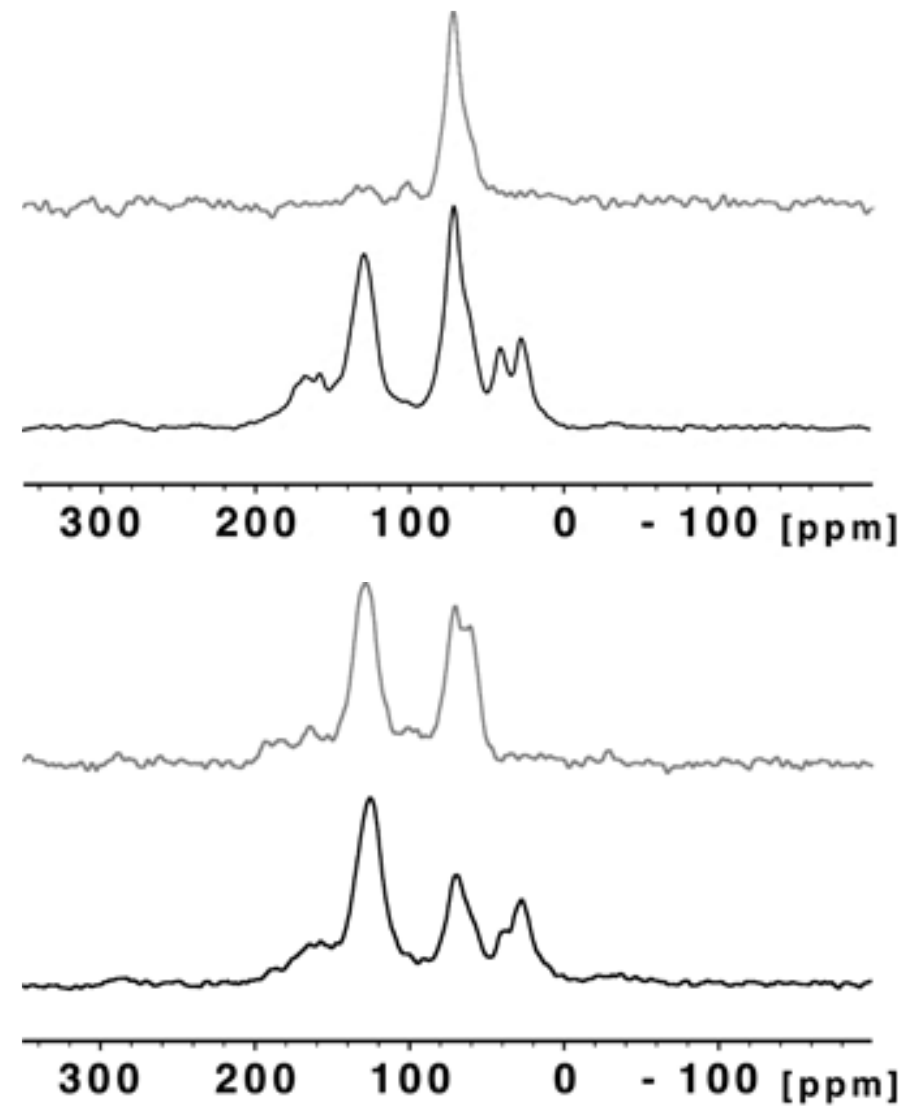

Figure 3. Qualitative CP (top) and quantitative (bottom) DP ${ }^{13} \mathrm{C}$ MAS NMR spectra of GO-A (grey) and GOA-OE-EST (black).

Based on a combination of characterization techniques, we efficiently proved the introduction of both chains on GO-A and we also demonstrated that the strategy is successful on different sources of GO. Nevertheless, the level of functionalization of GO-A was lower compared to GO-N, pointing out that various sources of GO can have different chemical reactivity. In addition, MAS NMR showed that GO-N and GO-A do not have exact composition (Table S3). In particular, in the ${ }^{13} \mathrm{C} \mathrm{CP}$ MAS NMR spectra the peak at $\sim 130 \mathrm{ppm}$ is less intense for GO-A compared to GO-N (Figure 3 and S8, top grey spectra), whereas in the quantitative ${ }^{13} \mathrm{C}$ DP MAS NMR spectra this peak is slightly more intense (45\%) as in the case of GO-N (37\%) (bottom grey spectra in Figure 3 and S8, Table S3). This result is indicative of a different distribution of protonated carbon atoms in both GO samples. ${ }^{[22]}$ The protonated carbon atoms seem to be homogeneously distributed on the surface of GO-N, thus the protons are able to transfer magnetization to the carbon atoms in close proximity. On the contrary, 
there seem to be fewer protonated carbon atoms, probably in the form of clusters, in the case of GOA. Therefore, only the protons at the cluster border are capable of transferring magnetization to the adjacent carbon atoms, which can explain the low intense peak at $\sim 130$ ppm for GO-A in comparison to GO-N in the CP spectra. This observation indicates that the localization of functional groups on GO surface is not homogeneous and can vary from one source to the other.

\section{Conclusion}

We have investigated three different strategies for the covalent double functionalization of GO through the chemoselective derivatization of the epoxides and the hydroxyl groups. The most efficient method appeared to be the combination of the opening of the epoxides and the esterification of the hydroxyls. A thorough characterization by different techniques including XPS, TGA, MAS NMR, and the Kaiser test confirmed the occurrence of the two successive reactions. The presence of amine functions allows further derivatization with molecules of interest in mild conditions such as amidation. The use of two orthogonally protected amino chains opens opportunities for the controlled derivatization of the amine functions with two different molecules via a selective and sequential cleavage of the orthogonal protecting groups. The multifunctionalized GO holds a high potential for future applications in different fields and in particular in nanomedicine where GO could be used as a multimodal platform for therapeutic and imaging applications.

\section{Experimental Section}

Materials and Methods are detailed in the SI, as well as the synthesis of compounds $\mathbf{4}$ and $\mathbf{5}$, protocols for the functionalization of GO, reaction schemes, and complementary characterization by the Kaiser test, XPS, TGA, TEM, and NMR.

\section{Acknowledgements}

This work was supported by the Centre National de la Recherche Scientifique (CNRS) and by the International Center for Frontier Research in Chemistry (icFRC). The authors gratefully acknowledge financial support from EU GRAPHENE Flagship project (no. 785219), from EU MSCA-RISE projet CARBO-IMmap (no. 734381) and from the Agence Nationale de la Recherche (ANR) through the LabEx project Chemistry of Complex Systems (ANR-10-LABX-0026_CSC). S.G. is indebted to the China Scholarship Council for supporting his PhD internship. We also wish to acknowledge C. Royer and V. Demais for help with TEM analyses at the Plateforme Imagerie in Vitro at the INCI (Strasbourg, France). 


\section{References}

[1] D. Chen, H. Feng, J. Li, Chem. Rev. 2012, 112, 6027-6053.

[2] P. Zheng, N. Wu, Chem. Asian J. 2017, 12, 2343-2353.

[3] Z. Gu, S. Zhu, L. Yan, F. Zhao, Y. Zhao, Adv. Mater. 2019, 31, 1800662.

[4] G. Reina, J. M. González-Domínguez, A. Criado, E. Vázquez, A. Bianco, M. Prato, Chem. Soc. Rev. 2017, 46, 4400-4416.

[5] D.-K. Ji, C. Ménard-Moyon, A. Bianco, Adv. Drug Deliv. Rev. 2019, 138, 211-232.

[6] G. Seber, J. Muñoz, S. Sandoval, C. Rovira, G. Tobias, M. Mas-Torrent, N. Crivillers, Adv. Mater. Interfaces 2018, 5, 1701072.

[7] B. Cai, S. Wang, L. Huang, Y. Ning, Z. Zhang, G.-J. Zhang, ACS Nano 2014, 8, 2632-2638.

[8] D. R. Dreyer, S. Park, C. W. Bielawski, R. S. Ruoff, Chem. Soc. Rev. 2010, 39, 228-240.

[9] D. R. Dreyer, A. D. Todd, C. W. Bielawski, Chem. Soc. Rev. 2014, 43, 5288-5301.

[10] S. Eigler, A. Hirsch, Angew. Chem. Int. Ed. 2014, 53, 7720-7738.

[11] V. Georgakilas, M. Otyepka, A. B. Bourlinos, V. Chandra, N. Kim, K. C. Kemp, P. Hobza, R. Zboril, K. S. Kim, Chem. Rev. 2012, 112, 6156-6214.

[12] I. A. Vacchi, C. Spinato, J. Raya, A. Bianco, C. Ménard-Moyon, Nanoscale 2016, 8, 1371413721.

[13] R. Imani, S. Prakash, H. Vali, S. Faghihi, Biomater. Sci. 2018, 6, 1636-1650.

[14] A. Rezaei, O. Akhavan, E. Hashemi, M. Shamsara, Chem. Mater. 2016, 28, 3004-3016.

[15] S. Luo, Z. Yang, X. Tan, Y. Wang, Y. Zeng, Y. Wang, C. Li, R. Li, C. Shi, ACS Appl. Mater. Interfaces 2016, 8, 17176-17186.

[16] K.-C. Mei, N. Rubio, P. M. Costa, H. Kafa, V. Abbate, F. Festy, S. S. Bansal, R. C. Hider, K. T. Al-Jamal, Chem. Commun. 2015, 51, 14981-14984.

[17] S. Guo, Y. Nishina, A. Bianco, C. Ménard-Moyon, Angew. Chem. Int. Ed., DOI: 10.1002/anie.201913461.

[18] P. Pérez-Martínez, J. M. Galvan-Miyoshi, J. Ortiz-López, J. Mater. Sci. 2016, 51, 10782-10792.

[19] S. Pei, H.-M. Cheng, Carbon 2012, 50, 3210-3228.

[20] M. Rogala, P. Dabrowski, P. J. Kowalczyk, I. Wlasny, W. Kozlowski, A. Busiakiewicz, I. Karaduman, L. Lipinska, J. M. Baranowski, Z. Klusek, Carbon 2016, 103, 235-241.

[21] A. M. Dimiev, L. B. Alemany, J. M. Tour, ACS Nano 2013, 7, 576-588.

[22] I. A. Vacchi, J. Raya, A. Bianco, C. Ménard-Moyon, 2D Mater. 2018, 5, 035037.

[23] S. Eigler, C. Dotzer, A. Hirsch, M. Enzelberger, P. Müller, Chem. Mater. 2012, 24, 1276-1282.

[24] S. Stankovich, D. A. Dikin, R. D. Piner, K. A. Kohlhaas, A. Kleinhammes, Y. Jia, Y. Wu, S. T. Nguyen, R. S. Ruoff, Carbon 2007, 45, 1558-1565. 
[25] J. I. Paredes, S. Villar-Rodil, A. Martínez-Alonso, J. M. D. Tascón, Langmuir 2008, 24, 1056010564.

[26] S. Kim, S. Zhou, Y. Hu, M. Acik, Y. J. Chabal, C. Berger, W. de Heer, A. Bongiorno, E. Riedo, Nature Mater. 2012, 11, 544-549.

[27] C. K. Chua, M. Pumera, Small 2015, 11, 1266-1272.

[28] P. V. Kumar, N. M. Bardhan, S. Tongay, J. Wu, A. M. Belcher, J. C. Grossman, Nature Chem. 2014, 6, 151-158.

[29] Z. Lin, Y. Yao, Z. Li, Y. Liu, Z. Li, C.-P. Wong, J. Phys. Chem. C 2010, 114, 14819-14825.

[30] J. T. Paci, T. Belytschko, G. C. Schatz, J. Phys. Chem. C 2007, 111, 18099-18111.

[31] M. C. Biesinger. X-ray Photoelectron Spectroscopy (XPS) Reference Pages. Available from: http://www.xpsfitting.com/

[32] J. C. Lascovich, R. Giorgi, S. Scaglione, Appl. Surf. Sci. 1991, 47, 17-21.

[33] A. J. Barlow, S. Popescu, K. Artyushkova, O. Scott, N. Sano, J. Hedley, P. J. Cumpson, Carbon 2016, 107, 190-197.

[34] A. Hunt, D. A. Dikin, E. Z. Kurmaev, T. D. Boyko, P. Bazylewski, G. S. Chang, A. Moewes, Adv. Funct. Mater. 2012, 22, 3950-3957.

[35] H. Pieper, C. E. Halbig, L. Kovbasyuk, M. R. Filipovic, S. Eigler, A. Mokhir, Chem. Eur. J. 2016, 22, 15389-15395.

[36] S. Zhou, A. Bongiorno, Sci. Rep. 2013, 3, 2484.

[37] K. Erickson, R. Erni, Z. Lee, N. Alem, W. Gannett, A. Zettl, Adv. Mater. 2010, 22, 4467-4472.

[38] C. G. Bochet, J. Chem. Soc., Perkin Trans. 1 2002, 125-142.

[39] M. Kessler, R. Glatthar, B. Giese, C. G. Bochet, Org. Lett. 2003, 5, 1179-1181.

[40] R. S. Givens, P. G. Conrad, A. L. Yousef, J.-III Lee, Photoremovable Protecting Groups, 2003. p. 2904. In: CRC Handbook of Organic Photochemistry and Photobiology (Eds.: W. M. Horspool, F. Lenci). 